\title{
Intrinsic association between diet and the gut microbiome: current evidence
}

\author{
This article was published in the following Dove Press journal: \\ Nutrition and Dietary Supplements \\ 7 October 2015 \\ Number of times this article has been viewed
}

\author{
Kathryn Winglee \\ Anthony A Fodor \\ Department of Bioinformatics and \\ Genomics, University of North \\ Carolina at Charlotte, Charlotte, \\ NC, USA
}

\begin{abstract}
The gut microbiome performs many crucial functions for the human host, but the molecular mechanisms by which host, microbe, and diet interact to mediate health and disease are only starting to be revealed. Here, we review the literature on how changes in the diet affect the microbiome. A number of studies have shown that within a geographic region, different diets (such as vegan vs omnivore) are associated with differences in a modest number of taxa, but do not reliably produce radical differences within the gut microbial community. In contrast, studies that look across continents consistently find profoundly different microbial communities between Westernized and traditional populations, although it remains unclear to what extent diet or other differences in lifestyle drive these distinct microbial community structures. Furthermore, studies that place subjects on controlled short-term experimental diets have found the resulting alterations to the gut microbial community to generally be small in scope, with changes that do not overcome initial individual differences in microbial community structure. These results emphasize that the human gut microbial community is relatively stable over time. In contrast, short-term changes in diet can cause large changes in metabolite profiles, including metabolites processed by the gut microbial community. These results suggest that commensal gut microbes have a great deal of genetic plasticity and can activate different metabolic pathways independent of changes to microbial community composition. Thus, future studies of how the diet impacts host health via the microbiome may wish to focus on functional assays such as transcriptomics and metabolomics, in addition to 16S rRNA and whole-genome metagenome shotgun analyses of DNA. Taken together, the literature is most consistent with a model in which the composition of the adult gut microbial community undergoes modest compositional changes in response to altered diet but can nonetheless respond very rapidly to dietary changes via up- or downregulation of metabolic pathways that can have profound and immediate consequences for host health.
\end{abstract}

Keywords: microbiome, transcriptomics, metabolomics, 16S rRNA, diet

\section{Introduction}

There is a growing body of evidence that the human microbiome, defined as the set of microorganisms that live on or in a person, is critically important to health. The human microbiome is extremely complex, with an estimated 10 microbial cells to every human cell in the body. ${ }^{1}$ These microorganisms encode 100 times the diversity of a single human genome, and, as a result, provide many crucial functions for their host. ${ }^{2}$ The microbiota has been shown to play a role in immune development and modulation, ${ }^{3-6}$ defense against pathogens, ${ }^{7-11}$ and food degradation. ${ }^{12}$

The key role our gut bacteria play in our nutrition has been particularly well studied. Bacteria help to break down and utilize nutrients our bodies would not otherwise be
Correspondence: Anthony A Fodor Department of Bioinformatics and Genomics, 920I University City Blvd, Charlotte, NC 28223, USA

Email anthony.fodor@gmail.com 
able to process. ${ }^{13}$ Among other critical functions, they assist with foraging nutrients, ${ }^{14}$ synthesizing vitamins, ${ }^{15,16}$ fermenting carbohydrates to generate short-chain fatty acids, ${ }^{17}$ bile acid metabolism, ${ }^{18}$ and regulating energy extraction and storage. ${ }^{19-21}$ Studies are now beginning to look in the other direction, at how diet and nutrition affect our bacteria and our health, and at whether dietary effects on health are mediated by the microbiome.

Dysbiosis of the gut microbiome has been linked with several metabolic diseases including diabetes, ${ }^{22}$ colorectal cancer, ${ }^{23-25}$ colorectal adenomas, ${ }^{26}$ inflammatory bowel disease, ${ }^{27-29}$ and obesity. ${ }^{30-32}$ When populations shift to a Westernized diet, the prevalence of these diseases markedly increases. ${ }^{33,34}$ However, it is unclear whether the microbial dysbiosis that may be associated with these diseases is a direct cause of disease or is simply part of the body's response to the disease. Part of the recent intense interest in the microbiome arises from the intriguing possibility that modulation of the bacterial community might alleviate disease risk associated with a Western diet or even reverse symptoms after disease onset.

The composition of the microbial community in each adult reflects the history of exposure to microbes, diet, drugs, and other environmental interactions. In the first few years of life, the gut microbiome is less distinguished from other microbes associated with the human host, but by age $2-3$, the infant gut is more similar to the adult gut with a composition at the phyla level usually dominated by a mix of Bacteroidetes and Firmicutes. ${ }^{35}$ The human adult gut microbiome is distinctively individual and distinctively stable. Numerous $16 \mathrm{~S}$ studies, including the human microbiome project, have noted a strong individual signature that is often largely stable over time..$^{36,37}$

Given the role of bacteria in human health and metabolism, and the potential to impact disease risk and progression by changing the microbiome, it is intriguing to consider the degree to which the adult human gut microbiota can be influenced by changes in lifestyle and diet. In the popular scientific literature, it is becoming an increasingly prevalent idea that changes in the microbiome induced by improved lifestyle can improve overall health. However, given the reported stability of the gut microbiome over time, it is unclear to what extent dietary manipulations can in fact alter the microbiome, how long a new diet would need to be adhered to in order to influence the microbiome, and whether such changes would themselves be permanent if the new diet were abandoned. Here we review the evidence in the literature describing how diet can change the microbiome and how the microbiome can at times display resilience in the face of dietary change. We find that while short-term changes in diet are robustly associated with changes in biomarkers of health outcomes and metabolic profiles, there are only modest changes to microbial community structure in response to diet change as measured by $16 \mathrm{~S}$-based sequencing surveys. While adult individuals with radically different lifestyles on different continents harbor very distinct microbiota, and the overall health benefits of improving diet are inarguable, it remains to be established that changes in diet can radically restructure the adult microbiota and that changes to microbial community composition are the direct cause of improved health outcomes from improved diet.

\section{Evolution of the gut microbiome in response to diet}

Several studies have analyzed the influence of diet on the microbiome in the context of evolution and development. Using 16S rRNA sequencing, Ley et $\mathrm{al}^{38}$ analyzed the fecal microbial community of 106 individuals representing 60 species. They found that community composition clustered both with phylogeny and host diet (carnivore, omnivore, or herbivore), with herbivores having the most diversity and humans clustering with other omnivores. This study suggests that the microbiome has evolved with its host in response to host diet. In a follow-up study analyzing shotgun sequences from 33 mammalian species, Muegge et $\mathrm{a}^{39}$ confirmed this finding and showed that the microbiota adapts to diet across lineages. Thus, these papers provide evidence that the gut microbial community structure is associated with diet across phylogeny, suggesting a strong interaction between what a host eats and its microbiome across a wide evolutionary space.

\section{Early effects of diet in infants}

Given that the microbiome has evolved with its host in response to diet, it is important to consider where the microbiome originates. Several studies have shown that the bacterial colonization of a baby begins at birth. Furthermore, it appears that there is a significant difference in the microbiota when comparing breastfed and formula-fed babies (reviewed by Collado et $\mathrm{a}^{40}$ ). A more recent study followed infants from birth to 12 months and showed progression of the microbiome with time, starting with differences in community composition based on the mode of birth ( $\mathrm{C}$-section compared to vaginally). ${ }^{41}$ Not only was a difference in the microbiome between breastfed and formula-fed babies observed, but also the cessation of breastfeeding was shown to have the largest effects on the microbiome, resulting in a community most 
similar to the mother. In fact, cessation of breastfeeding had a larger effect than the introduction of solid food. It is unclear what effects these differing communities may have later in life, but it seems that the influences of diet begin early.

\section{Effects of specific diets in animal models}

Animal models are a powerful tool to specifically look at the effects of a particular diet, as it is possible to precisely control their food intake. Furthermore, using gnotobiotic mice, it is possible to control the starting microbial community, eliminating the variation seen between individual humans. In fact, using gnotobiotic mice introduced to a 10 -member community of sequenced human gut bacteria and providing a diet in which the amount of macronutrients (protein, fat, polysaccharide, and simple sugar) were controlled, Faith et $\mathrm{al}^{42}$ were able to develop a linear model that could predict how the microbiome would respond to changes in the diet. This model predicted more than $60 \%$ of the species variation between diets.

In addition, several studies have analyzed the effects of a high-fat Western diet on the microbiota of mice. In a study comparing mice on a low-fat diet to mice on a high-fat Western diet, Turnbaugh et $\mathrm{al}^{43}$ found the microbiome clustered by diet. The Western diet was characterized by high abundance of Firmicutes, which was dominated by a bloom of Mollicutes, and low abundance of Bacteroidetes. By reducing either fat or carbohydrates in the Western diet, they found an association between weight and the reduction of Mollicutes abundance with an increase in Bacteroidetes. Additionally, they found that fructose and mannose metabolism and phosphotransferase systems increased with the Western diet while cell motility decreased. Thus, in mice, the amount of fat in the diet appears to affect the microbial composition.

These results were confirmed by Hildebrandt et al, ${ }^{44}$ who compared wild-type and RELM $\beta$ knockout mice on a high- or low-fat diet. Similar to Turnbaugh et al, ${ }^{43}$ they found that microbial composition clustered by genotype and diet, and they observed that a high-fat diet results in increased abundances of Firmicutes class Clostridiales and Delta-Proteobacteria and decreased abundances of Bacteroidetes class Bacteroidales compared to a low-fat diet. They also saw an increase in Mollicutes, but not the large bloom identified by Turnbaugh et al. ${ }^{43}$ Also similar to the study by Turnbaugh et al, ${ }^{43}$ these results were confirmed with whole-genome sequencing, which they also used to find decreases in amino acid and carbohydrate metabolism and increases in signal transduction and membrane transport (particularly ABC transporters) upon switching to the highfat diet. ${ }^{44}$ Furthermore, this increase in Firmicutes at the expense of Bacteroidetes on a Western diet, and the increase in transporters, was also found in gnotobiotic humanized mice containing microbiota transplanted from human stool. ${ }^{45}$ Several other studies have since confirmed these findings. ${ }^{46,47}$ Interestingly, some Bacteroidetes transplanted from lean humans into gnotobiotic mice appear to be able to invade the microbiome of mice with a human obese community composition, preventing weight gain. ${ }^{48}$ This suggests a direct and reversible correlation between Bacteroidetes and obesity. However, the invasion and protection from increased body mass was diet-dependent and only occurred on the low-fat diet and not high-fat diet. One caveat to many of these studies is that they have not ruled out a caging effect, which can result in strong confounding effects. ${ }^{49}$ Nevertheless, these studies provide compelling evidence that, at least in mice, high levels of fat in the diet can affect microbial community composition, particularly increased Firmicutes and decreased Bacteroidetes, which in turn results in alterations in carbohydrate metabolism and transport.

Similar studies have been performed analyzing the effects of fiber on the murine microbiome. For example, it appears that the additional fiber in the diet decreases Firmicutes while increasing Bacteroidetes, ${ }^{50-53}$ opposite the effects seen with fat. These effects are often tied to levels of short-chain fatty acids, which play a role in protection from pathogenic bacteria, provide energy to the host, and protect against gut inflammation, cancer, and weight gain. ${ }^{54-56}$ Other groups have shown similar effects in rats ${ }^{57-59}$ and pigs ${ }^{60}$ Furthermore, these changes appear to be correlated to changes in the metabolome. ${ }^{61}$ Given the beneficial effects of dietary fiber compared to fat, and the fact that they have opposing effects on the microbiome, decreasing the number of Firmicutes compared to Bacteroidetes appears beneficial to the host.

\section{Effect of specific diets in human trials}

Animal models provide valuable insights into the effects of diet on the microbiome, but they cannot replace human studies. Several groups have studied dietary effects in humans, often confirming the results of studies performed in other mammals, although often the interindividual variation between humans is stronger than the diet effect, unlike in animal models, which have controlled environments with low interindividual variation. One study focusing on 14 overweight men found no diet effect on the ratio of Firmicutes to Bacteroidetes when using a diet containing resistant starch, 
nonstarch polysaccharides, or reduced carbohydrates, ${ }^{62}$ in contrast to the animal studies discussed above. However, they did identify changes at lower taxonomic levels, such as increased Eubacterium rectale and Ruminococcus bromii on diets with resistant starch and decreased Collinsella aerofaciens with the low-carbohydrate weight loss diet. ${ }^{62}$ Although the small sample size limits the interpretation of these findings, they have been confirmed by other groups, which have also observed a decrease in E. rectale, Bifidobacterium, and Roseburia upon decreasing carbohydrate in the diet. ${ }^{63-68}$

Another aspect of the diet that has also been studied in detail in both mice and humans is L-carnitine, which is present in high abundance in red meat. ${ }^{69}$ This study showed that the amount of L-carnitine in the diet affects some members of the microbiome and leads to increases in atherosclerosis through trimethylamine- $N$-oxide synthesis. Intriguingly, the long-term dietary habits of the subject affected trimethylamine- $N$-oxide synthesis, and the risk of cardiovascular disease was affected by antibiotics and the composition of the microbiome, suggesting a direct link between diet, microbial composition, and disease.

Another study looked at the effects of a plant-based diet compared to an animal-based diet and found changes in $\beta$-diversity in the animal but not plant diet, with the animal diet resulting in an increase in Bilophila and Bacteroidetes (Alistipes and Bacteroides) and a decrease in Firmicutes (Roseburia, Eubacterium rectale, and Ruminococcus bromii)..$^{70}$ There was also evidence of bacteria from food, particularly cheese and cured meats, surviving the transit to the gut, suggesting one possible mechanism for the observed changes to the microbial community. As in other studies of the response to the gut microbiota to a short-term change in diet (discussed later), the differences in microbial community composition observed in response to diet change were smaller than differences in microbial community composition between subjects, and the individual signature of the microbial community persisted longitudinally across time despite the change in diet. In terms of gene expression, subjects on the animal diet had higher levels of vitamin biosynthesis, polycyclic aromatic hydrocarbon degradation, and $\beta$-lactamases. Importantly, these differences in gene expression became established in response to the 5-day experimental diet, suggesting a rapid response in the transcriptome in response to dietary changes. Thus, in humans, it appears that although diet affects the relative abundance of a few genera, overall these changes are small compared to the variation between individuals, although changes in gene expression occur rapidly.
A number of other studies have also reported rapid, modest changes to the microbiome in response to a controlled experimental diet. For example, Spencer et $\mathrm{al}^{71}$ found little change in microbial composition when moving subjects from their normal diet to a low-choline experimental diet, after a small initial change in microbial community composition observed in the first 24 hours. In a separate controlled feeding experiment, Wu et $\mathrm{al}^{72}$ also observed rapid (24-hour) changes in the gut microbiome, although again the modest shortterm changes that were observed were also not sufficient to overcome intersubject variation. Additionally, Wu et $\mathrm{al}^{73}$ found very little difference in microbial composition when comparing vegans to omnivores, despite pronounced differences in the plasma metabolome. In contrast to the $\mathrm{Wu}$ et $\mathrm{al}^{73}$ study, several other groups have identified differences in the microbiome between vegans or vegetarians and omnivores, although these differences have generally been modest with only a few specific organisms identified as statistically different between vegans and nonvegans. ${ }^{69,74}$ Most of these studies did not examine other factors, such as smoking, which are also known to affect the microbiome, and which could also cause some of the differences between studies. Nonetheless, while it appears that very different diets may produce small but consistent differences in the microbial community, the literature is most consistent with the hypothesis that radically different diets do not radically restructure the microbial community in adults in the same geographic region. Presumably, therefore, other factors such as childhood exposure to different microbes, childhood diets, exposure to antibiotics, host genetics, disease history, exposure to different physical environments, etc explain the distinct gut microbial community structures across different individuals.

\section{Association between microbiome, lifestyle, and diet}

As societies become more developed and move away from traditional lifestyles, many factors change, including the diet and the microbiome of individuals within that society. For better or worse, these changes become an intrinsic part of that culture. In contrast to studies within a geographic region, which tend to show only modest clustering of the microbial community with diet, numerous studies have shown that people on different continents with extremely different lifestyles have distinct microbial communities. For example, one study found significant differences in the microbiome when comparing children 1-6 years of age from either rural villages in Burkina Faso or from Florence, Italy. ${ }^{54}$ Children from Burkina Faso were enriched 
for Bacteroidetes at the expense of Firmicutes, and had increased Prevotella and Xylanibacter but decreased Enterobacteriaceae. Interestingly, the only samples that did not cluster separately were the youngest children, who were still breastfeeding. These samples were intermediate between the others, suggesting that variation in diet after weaning may be one of the biggest contributors driving individual differences in the microbial community. This overall change in the ratio of Firmicutes to Bacteroidetes with Westernization is in line with the studies above that found similar changes with high-fat diets.

In a later study, Yatsunenko et $\mathrm{al}^{35}$ confirmed the finding of differences between Western and African communities when analyzing the microbiome of Americans, Amerindians from Venezuela, and Malawians across a larger age range (0-70 years). These differences were most pronounced when comparing Americans to the other two groups, who have a diet dominated by corn and cassava as opposed to the proteinrich diet of Americans. Importantly, these changes were seen even in children less than 3 years of age, emphasizing how early these effects begin. The ability to differentiate the microbiome of Western individuals and individuals from other cultures has also been seen in other studies, and even between different European countries. ${ }^{75-78}$

A weakness of all association studies is that many other factors besides diet, such as antibiotic exposure or exposure to a rural compared to an urban environment, may explain the differences observed in the microbiome between Westernized city dwellers and rural cohorts living traditional lifestyles. In a recent experiment that directly addressed the effects of short-term changes in diet, $\mathrm{O}^{\prime}$ Keefe et $\mathrm{al}^{79}$ utilized a crossover design in which they fed African-Americans a traditional African diet and rural Africans a Westernized diet. Intriguingly, they found that biomarkers of colon cancer, including proliferative rates as well as immunohistochemical markers of inflammation, were substantially altered by this 2 week change of diet, with the Western diet increasing the markers of risk and the traditional diet decreasing the markers of risk. O'Keefe et al ${ }^{79}$ demonstrated that these changes are mediated by the activity of the gut microbial community, with the traditional diet promoting beneficial butyrogenesis and the Western diet promoting harmful secondary bile acids. However, despite these changes in host health with diet exchange, most likely propagated via the microbial community, O'Keefe et $\mathrm{al}^{79}$ report that "the compositional changes that were specifically associated to the diet switch were minor". This pattern is similar to the studies by $\mathrm{Wu}$ et $\mathrm{al}^{72}$ and Spencer et al, ${ }^{71}$ which also reported minor changes to the microbial community on exposure to a new diet followed by a period of stability.

Taken together, these studies suggest that once the microbial community in adults is established, changes in diet may not be enough to completely restructure the microbial community. A natural question to ask is to what overall degree the adult gut microbial community is stable. Are there any changes to diet or lifestyle that can overcome individual differences in the gut microbial community? Or, by contrast, once the adult gut microbial community is set, is it essentially impossible to change through dietary or lifestyle modifications? One intriguing study suggests that the microbial community in adults can in fact be changed with a radical change in lifestyle with a time course on the order of a year; Claesson et $\mathrm{al}^{80}$ analyzed the microbiota of elderly ( $>65$ year old) Irish individuals in different residence settings. They found that subjects who were in long-term care for over a year had a largely distinct microbial community from city residents. Intriguingly, subjects who had been in the nursing home for 6 weeks had a microbial community intermediate between city residents and residents of the nursing home who had been there for over a year. While many other factors besides diet could explain these temporal variances, these data are consistent with a model in which lifestyle changes must be applied for a period of around 1 year before the microbial community completely responds. However, there is an urgent research need to separate the effects of diet form other possible influences shaping the microbial community over this time scale, although these are difficult experiments to perform, as controlled dietary studies that last a year are outside the budget of many standard grant applications.

\section{Conclusion}

It is clear that even short-term changes in diet can have immediate and positive effects on host health. A Westernized diet, with its emphasis on simple sugars and animal proteins and fats, is inarguably a risk factor for a number of metabolic diseases. Even in a period of as little as 2 weeks, switching from a Westernized diet to a traditional African diet produced improvement in biomarkers of risk for colorectal cancer. ${ }^{79}$ Numerous studies have reported, however, that short-term changes in diet, by and large, produce changes in the gut microbiome that are modest when compared to interindividual differences. ${ }^{65,71-73}$ Thus, although large changes in response to diet are seen in the controlled setting of animal models, which have little interindividual variation, these alterations in the microbiome are less distinct in 
humans undergoing short-term dietary changes. Although longer term controlled diet studies are urgently needed, the balance of evidence in the literature suggests that it may take as long as a year for the gut microbiome to fully respond to even radical changes in lifestyle.

One of the findings of the Human Microbiome Project was that the gene profiles of microbes, derived from whole-genome shotgun sequencing, are much more constant across subjects than the taxonomic profiles derived from 16S rRNA sequencing. ${ }^{37}$ This observation is consistent with results that were identified in the African and American diet crossover study, which found substantial evidence for microbiome-mediated changes to metabolites that were independent of large changes to gut microbial community composition. ${ }^{79}$ These data are consistent with a model in which many different gut commensal bacteria have the genetic potential to perform activities that are either beneficial or harmful to the host. Under this model, the fuels we provide to these organisms determine whether the "good" or "bad" genetic potential of the microbiome is realized regardless of the taxonomic makeup of gut commensal organisms. If this is true, research programs that emphasize RNA-seq and microbial transcriptomics may be more informative than experiments which emphasize $16 \mathrm{~S}$ or whole-genome shotgun sequencing in revealing how diet-microbial interactions impact host health.

Urgent questions in need of further research include: 1) Are the modest changes that occur within a day or two of exposure to a new diet biologically important and do they contribute to health effects in the host? 2) In a controlled feeding experiment in which subjects were placed on identical diets for a period of a year or more, would the microbiome of these subjects converge, overwhelming initial individual differences? Or do factors other than diet structure the microbial community at this time scale? 3) Even if this convergence occurred, would it be biologically significant in providing beneficial effects to the host upon new exposure to an improved diet? Answering these questions is especially relevant as more people migrate and become introduced to a Westernized lifestyle.

These questions are particularly of concern as companies continue to advertise products meant to alter the microbiome, such as prebiotics and probiotics. Probiotics are organisms or substances that improve the microbial balance while prebiotics are substances that simulate the growth or activity of bacteria already present..$^{81,82}$ The effectiveness of probiotics and prebiotics has been reviewed in detail elsewhere, ${ }^{83,84}$ and is outside the scope of this paper. It seems clear, however, that even if certain substances can affect the relative abundance of specific organisms, the effects of such changes on host health in general have not been established. If it turns out that the activation of pathways within commensals matters more than the identity of particular commensals, probiotics may have limited effectiveness in impacting human health.

Ultimately, there are two models of how the microbiome can impact host health and we have as yet insufficient data to resolve these two models. In the first model there are "good bacteria", such as the microbes that exist in rural African communities, and "bad bacteria" such as the microorganisms that exist in a typical American. Under this model, replacing "bad bacteria" with "good bacteria" would improve health even without changes in diet. Under the second model, microorganisms have the potential to be either "good" or "bad" and the genes that are expressed by bacteria depend on the diet the host consumes. Under this model, bacteria have the potential to be friend or foe, and changing the microbial composition would not have as large an impact on host health as long-term changes in diet that alter microbial gene expression. These models are not mutually exclusive, and it could also be possible that the beneficial effects of altered diet results from changes in both microbial community composition and gene expression.

While more research will be required to work out the mechanisms by which host, diet, and microbes interact to produce health phenotypes, it is clear that those wishing to enjoy good health can make dietary choices limiting exposure to meats, fats, and simple sugars. Whether this changes the microbiome and therefore promotes health or just encourages whatever microbiome we may have already acquired to perform more beneficial functions, there is overwhelming support for the hypothesis that good choices in controlling the diet side of host-microbial interactions can make substantial contributions in promoting host health.

\section{Disclosure}

Anthony A Fodor has consulted for the Salix Corporation. The authors report no other conflicts of interest in this work.

\section{References}

1. Gill SR, Pop M, Deboy RT, et al. Metagenomic analysis of the human distal gut microbiome. Science. 2006;312(5778):1355-1359.

2. Xu J, Bjursell MK, Himrod J, et al. A genomic view of the humanBacteroides thetaiotaomicron symbiosis. Science. 2003;299(5615): 2074-2076.

3. Nishio J, Honda K. Immunoregulation by the gut microbiota. Cell Mol Life Sci. 2012;69(21):3635-3650.

4. Hooper LV, Littman DR, Macpherson AJ. Interactions between the microbiota and the immune system. Science. 2012;336(6086):1268-1273.

5. Maynard CL, Elson CO, Hatton RD, Weaver CT. Reciprocal interactions of the intestinal microbiota and immune system. Nature. 2012;489(7415):231-241. 
6. Jarchum I, Pamer EG. Regulation of innate and adaptive immunity by the commensal microbiota. Curr Opin Immunol. 2011;23(3): 353-360.

7. Wilks J, Golovkina T. Influence of microbiota on viral infections. PLoS Pathog. 2012;8(5):e1002681.

8. Kuss SK, Best GT, Etheredge CA, et al. Intestinal microbiota promote enteric virus replication and systemic pathogenesis. Science. 2011;334(6053):249-252.

9. Sassone-Corsi M, Raffatellu M. No vacancy: how beneficial microbes cooperate with immunity to provide colonization resistance to pathogens. J Immunol. 2015;194(9):4081-4087.

10. Kamada N, Kim YG, Sham HP, et al. Regulated virulence controls the ability of a pathogen to compete with the gut microbiota. Science. 2012;336(6086):1325-1329.

11. Sekirov I, Finlay BB. The role of the intestinal microbiota in enteric infection. J Physiol. 2009;587(Pt 17):4159-4167.

12. Sharon G, Garg N, Debelius J, Knight R, Dorrestein PC, Mazmanian SK. Specialized metabolites from the microbiome in health and disease. Cell Metab. 2014;20(5):719-730.

13. Hooper LV, Midtvedt T, Gordon JI. How host-microbial interactions shape the nutrient environment of the mammalian intestine. Annu Rev Nutr. 2002;22:283-307.

14. Sonnenburg JL, Xu J, Leip DD, et al. Glycan foraging in vivo by an intestine-adapted bacterial symbiont. Science. 2005;307(5717): 1955-1959.

15. Ottman N, Smidt H, de Vos WM, Belzer C. The function of our microbiota: who is out there and what do they do? Front Cell Infect Microbiol. 2012;2:104.

16. Said HM. Intestinal absorption of water-soluble vitamins in health and disease. Biochem J. 2011;437(3):357-372.

17. Louis P, Duncan SH, McCrae SI, Millar J, Jackson MS, Flint HJ. Restricted distribution of the butyrate kinase pathway among butyrate-producing bacteria from the human colon. J Bacteriol. 2004;186(7):2099-2106.

18. Brestoff JR, Artis D. Commensal bacteria at the interface of host metabolism and the immune system. Nat Immunol. 2013;14(7):676-684.

19. Wostmann BS, Larkin C, Moriarty A, Bruckner-Kardoss E. Dietary intake, energy metabolism, and excretory losses of adult male germfree Wistar rats. Lab Anim Sci. 1983;33(1):46-50.

20. Backhed F, Ding H, Wang T, et al. The gut microbiota as an environmental factor that regulates fat storage. Proc Natl Acad Sci US A. 2004;101(44):15718-15723.

21. Turnbaugh PJ, Ley RE, Mahowald MA, Magrini V, Mardis ER, Gordon JI. An obesity-associated gut microbiome with increased capacity for energy harvest. Nature. 2006;444(7122):1027-1031.

22. Qin J, Li Y, Cai Z, et al. A metagenome-wide association study of gut microbiota in type 2 diabetes. Nature. 2012;490(7418):55-60.

23. Louis P, Hold GL, Flint HJ. The gut microbiota, bacterial metabolites and colorectal cancer. Nat Rev Microbiol. 2014;12(10):661-672.

24. Arthur JC, Gharaibeh RZ, Muhlbauer M, et al. Microbial genomic analysis reveals the essential role of inflammation in bacteria-induced colorectal cancer. Nat Commun. 2014;5:4724.

25. Schwabe RF, Jobin C. The microbiome and cancer. Nat Rev Cancer. 2013;13(11):800-812.

26. Sanapareddy N, Legge RM, Jovov B, et al. Increased rectal microbial richness is associated with the presence of colorectal adenomas in humans. ISME J. 2012;6(10):1858-1868.

27. Qin J, Li R, Raes J, et al. A human gut microbial gene catalogue established by metagenomic sequencing. Nature. 2010;464(7285):59-65.

28. Ott SJ, Musfeldt M, Wenderoth DF, et al. Reduction in diversity of the colonic mucosa associated bacterial microflora in patients with active inflammatory bowel disease. Gut. 2004;53(5):685-693.

29. Kostic AD, Xavier RJ, Gevers D. The microbiome in inflammatory bowel disease: current status and the future ahead. Gastroenterology 2014;146(6):1489-1499.

30. Turnbaugh PJ, Hamady M, Yatsunenko T, et al. A core gut microbiome in obese and lean twins. Nature. 2009;457(7228):480-484.
31. Janssen AW, Kersten S. The role of the gut microbiota in metabolic health. FASEB J. 2015;29(8):3111-3123.

32. Cani PD, Bibiloni R, Knauf C, et al. Changes in gut microbiota control metabolic endotoxemia-induced inflammation in high-fat diet-induced obesity and diabetes in mice. Diabetes. 2008;57(6):1470-1481.

33. Mokdad AH, Ford ES, Bowman BA, et al. Prevalence of obesity, diabetes, and obesity-related health risk factors, 2001. JAMA. 2003; 289(1):76-79.

34. Kahn SE, Hull RL, Utzschneider KM. Mechanisms linking obesity to insulin resistance and type 2 diabetes. Nature. 2006;444(7121):840-846.

35. Yatsunenko T, Rey FE, Manary MJ, et al. Human gut microbiome viewed across age and geography. Nature. 2012;486(7402):222-227.

36. Ding T, Schloss PD. Dynamics and associations of microbial community types across the human body. Nature. 2014;509(7500):357-360.

37. Human Microbiome Project C. Structure, function and diversity of the healthy human microbiome. Nature. 2012;486(7402):207-214.

38. Ley RE, Hamady M, Lozupone C, et al. Evolution of mammals and their gut microbes. Science. 2008;320(5883):1647-1651.

39. Muegge BD, Kuczynski J, Knights D, et al. Diet drives convergence in gut microbiome functions across mammalian phylogeny and within humans. Science. 2011;332(6032):970-974.

40. Collado MC, Cernada M, Bauerl C, Vento M, Perez-Martinez G. Microbial ecology and host-microbiota interactions during early life stages. Gut Microbes. 2012;3(4):352-365.

41. Backhed F, Roswall J, Peng Y, et al. Dynamics and stabilization of the human gut microbiome during the first year of life. Cell Host Microbe. 2015;17(5):690-703.

42. Faith JJ, McNulty NP, Rey FE, Gordon JI. Predicting a human gut microbiota's response to diet in gnotobiotic mice. Science. 2011;333(6038):101-104.

43. Turnbaugh PJ, Backhed F, Fulton L, Gordon JI. Diet-induced obesity is linked to marked but reversible alterations in the mouse distal gut microbiome. Cell Host Microbe. 2008;3(4):213-223.

44. Hildebrandt MA, Hoffmann C, Sherrill-Mix SA, et al. High-fat diet determines the composition of the murine gut microbiome independently of obesity. Gastroenterology. 2009;137(5):1716-1724; e1711-e1712.

45. Turnbaugh PJ, Ridaura VK, Faith JJ, Rey FE, Knight R, Gordon JI. The effect of diet on the human gut microbiome: a metagenomic analysis in humanized gnotobiotic mice. Sci Transl Med. 2009;1(6):6ra14.

46. Everard A, Lazarevic V, Gaia N, et al. Microbiome of prebiotic-treated mice reveals novel targets involved in host response during obesity. ISME J. 2014;8(10):2116-2130.

47. Siddharth J, Holway N, Parkinson SJ. A Western diet ecological module identified from the 'humanized' mouse microbiota predicts diet in adults and formula feeding in children. PLoS One. 2013;8(12):e83689.

48. Ridaura VK, Faith JJ, Rey FE, et al. Gut microbiota from twins discordant for obesity modulate metabolism in mice. Science. 2013;341(6150):1241214

49. McCafferty J, Muhlbauer M, Gharaibeh RZ, et al. Stochastic changes over time and not founder effects drive cage effects in microbial community assembly in a mouse model. ISME J. 2013;7(11):2116-2125.

50. Cox LM, Cho I, Young SA, et al. The nonfermentable dietary fiber hydroxypropyl methylcellulose modulates intestinal microbiota. FASEB J. 2013;27(2):692-702.

51. Tachon S, Zhou J, Keenan M, Martin R, Marco ML. The intestinal microbiota in aged mice is modulated by dietary resistant starch and correlated with improvements in host responses. FEMS Microbiol Ecol. 2013;83(2):299-309.

52. Berger K, Falck P, Linninge $\mathrm{C}$, et al. Cereal byproducts have prebiotic potential in mice fed a high-fat diet. J Agric Food Chem. 2014;62(32):8169-8178.

53. Trompette A, Gollwitzer ES, Yadava K, et al. Gut microbiota metabolism of dietary fiber influences allergic airway disease and hematopoiesis. Nat Med. 2014;20(2):159-166.

54. De Filippo C, Cavalieri D, Di Paola M, et al. Impact of diet in shaping gut microbiota revealed by a comparative study in children from Europe and rural Africa. Proc Natl Acad Sci U S A. 2010;107(33):14691-14696. 
55. Lin HV, Frassetto A, Kowalik EJ Jr, et al. Butyrate and propionate protect against diet-induced obesity and regulate gut hormones via free fatty acid receptor 3-independent mechanisms. PLoS One. 2012;7(4):e35240.

56. Scheppach W, Weiler F. The butyrate story: old wine in new bottles? Curr Opin Clin Nutr Metab Care. 2004;7(5):563-567.

57. Jakobsdottir G, Xu J, Molin G, Ahrne S, Nyman M. High-fat diet reduces the formation of butyrate, but increases succinate, inflammation, liver fat and cholesterol in rats, while dietary fibre counteracts these effects. PLoS One. 2013;8(11):e80476.

58. Fak F, Jakobsdottir G, Kulcinskaja E, et al. The physico-chemical properties of dietary fibre determine metabolic responses, short-chain fatty acid profiles and gut microbiota composition in rats fed low- and high-fat diets. PLoS One. 2015;10(5):e0127252.

59. Kleessen B, Stoof G, Proll J, Schmiedl D, Noack J, Blaut M. Feeding resistant starch affects fecal and cecal microflora and short-chain fatty acids in rats. J Anim Sci. 1997;75(9):2453-2462.

60. Umu OC, Frank JA, Fangel JU, et al. Resistant starch diet induces change in the swine microbiome and a predominance of beneficial bacterial populations. Microbiome. 2015;3:16.

61. Marcobal A, Kashyap PC, Nelson TA, et al. A metabolomic view of how the human gut microbiota impacts the host metabolome using humanized and gnotobiotic mice. ISME J. 2013;7(10):1933-1943.

62. Walker AW, Ince J, Duncan $\mathrm{SH}$, et al. Dominant and diet-responsive groups of bacteria within the human colonic microbiota. ISME J. 2011;5(2):220-230.

63. Duncan SH, Belenguer A, Holtrop G, Johnstone AM, Flint HJ, Lobley GE. Reduced dietary intake of carbohydrates by obese subjects results in decreased concentrations of butyrate and butyrate-producing bacteria in feces. Appl Environ Microbiol. 2007;73(4):1073-1078.

64. Duncan SH, Lobley GE, Holtrop G, et al. Human colonic microbiota associated with diet, obesity and weight loss. Int $J$ Obes (Lond). 2008;32(11):1720-1724.

65. Cotillard A, Kennedy SP, Kong LC, et al. Dietary intervention impact on gut microbial gene richness. Nature. 2013;500(7464):585-588.

66. Brinkworth GD, Noakes M, Clifton PM, Bird AR. Comparative effects of very low-carbohydrate, high-fat and high-carbohydrate, low-fat weight-loss diets on bowel habit and faecal short-chain fatty acids and bacterial populations. Br J Nutr. 2009;101(10):1493-1502.

67. Holscher HD, Caporaso JG, Hooda S, Brulc JM, Fahey GC Jr, Swanson KS. Fiber supplementation influences phylogenetic structure and functional capacity of the human intestinal microbiome: follow-up of a randomized controlled trial. Am J Clin Nutr. 2015;101(1):55-64.

68. Russell WR, Gratz SW, Duncan SH, et al. High-protein, reducedcarbohydrate weight-loss diets promote metabolite profiles likely to be detrimental to colonic health. Am J Clin Nutr. 2011;93(5): 1062-1072.
69. Koeth RA, Wang Z, Levison BS, et al. Intestinal microbiota metabolism of L-carnitine, a nutrient in red meat, promotes atherosclerosis. Nat Med. 2013;19(5):576-585.

70. David LA, Maurice CF, Carmody RN, et al. Diet rapidly and reproducibly alters the human gut microbiome. Nature. 2014;505(7484):559-563.

71. Spencer MD, Hamp TJ, Reid RW, Fischer LM, Zeisel SH, Fodor AA. Association between composition of the human gastrointestinal microbiome and development of fatty liver with choline deficiency. Gastroenterology. 2011;140(3):976-986.

72. Wu GD, Chen J, Hoffmann C, et al. Linking long-term dietary patterns with gut microbial enterotypes. Science. 2011;334(6052):105-108.

73. Wu GD, Compher C, Chen EZ, et al. Comparative metabolomics in vegans and omnivores reveal constraints on diet-dependent gut microbiota metabolite production. Gut. Epub 2014 Nov 26.

74. Zimmer J, Lange B, Frick JS, et al. A vegan or vegetarian diet substantially alters the human colonic faecal microbiota. Eur J Clin Nutr. 2012;66(1):53-60.

75. Mueller S, Saunier K, Hanisch C, et al. Differences in fecal microbiota in different European study populations in relation to age, gender, and country: a cross-sectional study. Appl Environ Microbiol. 2006;72(2):1027-1033.

76. Ou J, Carbonero F, Zoetendal EG, et al. Diet, microbiota, and microbial metabolites in colon cancer risk in rural Africans and African Americans. Am J Clin Nutr. 2013;98(1):111-120.

77. Grzeskowiak L, Collado MC, Mangani C, et al. Distinct gut microbiota in southeastern African and northern European infants. J Pediatr Gastroenterol Nutr. 2012;54(6):812-816.

78. Lin A, Bik EM, Costello EK, et al. Distinct distal gut microbiome diversity and composition in healthy children from Bangladesh and the United States. PLoS One. 2013;8(1):e53838.

79. O'Keefe SJ, Li JV, Lahti L, et al. Fat, fibre and cancer risk in African Americans and rural Africans. Nat Commun. 2015;6:6342.

80. Claesson MJ, Jeffery IB, Conde S, et al. Gut microbiota composition correlates with diet and health in the elderly. Nature. 2012;488(7410):178-184.

81. Gibson GR, Roberfroid MB. Dietary modulation of the human colonic microbiota: introducing the concept of prebiotics. J Nutr. 1995;125(6):1401-1412.

82. Bindels LB, Delzenne NM, Cani PD, Walter J. Towards a more comprehensive concept for prebiotics. Nat Rev Gastroenterol Hepatol. 2015;12(5):303-310.

83. Patel R, DuPont HL. New approaches for bacteriotherapy: prebiotics, new-generation probiotics, and synbiotics. Clin Infect Dis. 2015;60(Suppl 2):S108-S121.

84. Scott KP, Antoine JM, Midtvedt T, van Hemert S. Manipulating the gut microbiota to maintain health and treat disease. Microb Ecol Health Dis. 2015;26:25877.
Nutrition and Dietary Supplements

\section{Publish your work in this journal}

Nutrition and Dietary Supplements is an international, peer-reviewed, open access journal focusing on research into nutritional requirements in health and disease, impact on metabolism and the identification and optimal use of dietary strategies and supplements necessary for normal growth and development. The journal welcomes papers covering
Dovepress

original research, basic science, clinical \& epidemiological studies, reviews and evaluations, guidelines, expert opinion and commentary, case reports and extended reports. The manuscript management system is completely online and includes a very quick and fair peer-review system, which is all easy to use. 